\title{
Vitamin C intake and periodontal disease
}

\author{
Grapefruit consumption improves vitamin $C$ status in periodontitis patients \\ H. Staudte, B. W. Sigusch and E. Glockmann Br Dent J 2005; 199: 213-217
}

\section{Objective}

Previous studies demonstrate a relationship between a lack of vitamin C and increased risk of periodontal disease. In the present study we examine the vitamin $C$ plasma levels and inflammatory measures in periodontitis patients before and after the consumption of grapefruit. Subjects and methods

Fifty-eight patients with chronic periodontitis were assigned to the test group (non-smokers $n=21$, smokers $n=17$ ) and a diseased control group (non-smokers $n=11$, smokers $n=9$ ). Furthermore, 22 healthy subjects were recruited to compare vitamin $\mathrm{C}$ plasma levels between periodontally diseased and healthy subjects. Clinical evaluations, including plaque index (PI), sulcus bleeding index (SBI), probing pocket depths (PPD) and plasma vitamin C levels, were performed at baseline, and after two weeks of grapefruit consumption.

Results

At baseline, we observed significantly reduced plasma vitamin C levels in the test group and diseased controls in comparison with the healthy controls. On principle, smokers showed lower levels of vitamin C (mean $0.39 \pm 0.17 \mathrm{mg} \mathrm{dl}^{-1}$ ) than non-smokers (mean $0.56 \pm 0.29 \mathrm{mg} \mathrm{dl}^{-1}$ ). After grapefruit consumption, the mean plasma vitamin C levels rose significantly in the test group compared to the diseased controls (nonsmokers: $0.87 \pm 0.39 \mathrm{mg} \mathrm{dl}^{-1}$, smokers: $0.74 \pm 0.30 \mathrm{mg} \mathrm{dl}^{-1}$ ). Furthermore the $\mathrm{SBI}$ was reduced in the test group (non-smokers: from 1.68 \pm 0.6 to $1.05 \pm 0.6, p<0.001)$, whereas PI and PPD were unaffected.

Conclusion

The present results show that periodontitis patients are characterised by plasma vitamin C levels below the normal range, especially in smokers. The intake of grapefruit leads to an increase in plasma vitamin C levels and improves sulcus bleeding scores. Longer term studies are necessary to determine whether other periodontal outcomes improve with such supplementation especially in smokers.

\section{IN BRIEF}

- Previous published data have suggested that a lack of vitamin C increases the risk of periodontal disease. This study shows that periodontitis patients, especially smokers, have significantly lower plasma vitamin $C$ levels than healthy subjects.

- Vitamin C is involved in immunological functions, eg phagocytosis, wound healing and shows antioxidant properties. These functions make it relevant for periodontal therapy and prevention.

- In this study the consumption of grapefruit improved the vitamin C supply in periodontitis patients and decreased the sulcus bleeding index. Possibly, the recommendation of vitamin $\mathrm{C}$ rich foods could support the management of periodontal disease.

\section{COMMENT}

In spite of intensive research in periodontology the number of periodontal diseases are very high worldwide. In dental practice we are often confronted with severe and therapy resistant forms of periodontitis. The use of mechanical and chemical treatments yield only momentary improvement of the periodontal conditions in some cases. Additional to local factors the investigation of physiological conditions influencing the periodontal system could also be important.

In this study, Staudte, Sigusch and Glockmann measured decreased plasma vitamin C levels in periodontitis patients, especially in smokers. To improve the vitamin $C$ supply they chose grapefruit as a natural source of vitamin C. In the progression of the study the patients ate two grapefruits per day for a period of two weeks. The investigation of the gingival inflammation and the plasma vitamin C levels showed an improvement after the grapefruit consumption, whereas the plaque index and the pocket depths were unaffected. The authors ascribed these results to the physiological functions of vitamin $\mathrm{C}$ in collagen synthesis, the immune and the antioxidant system. Furthermore they supposed that other biological substances in grapefruit may also have positive effects.

The contents of the study allude to a topical field in periodontal research. The involvement of environmental factors, eg nutrition, in the choice of the periodontal therapy is important for successful and durable healing. In earlier times vitamin $\mathrm{C}$ was the object of experimental periodontal investigations, but the results were often contradictory. This clinical study shows the positive effects of vitamin $\mathrm{C}$ rich fruits on periodontal health, and deliver a possibility to complement periodontal therapy. In their study the authors used grapefruits as a source of vitamin C. However grapefruits and all the other citrus fruits may lead to dental erosion by frequent consumption. In this case it is necessary to consider other sources of vitamin C like green vegetables which do not have an erosive effect on the hard tissues of teeth.

In the future more controlled studies are needed to assess the influence of specific nutrients on periodontal disease onset, progression and treatment.

A. Borutta, Director of the WHO Collaboration Centre, Prevention of oral diseases

doi: 10.1038/sj.bdj.4812616 